\title{
La incorporación de las TIC y su aporte en la interacción entre ciudadano y policía en la gestión de la información*
}

\author{
The incorporation of ICT and its contribution in the interaction between citizen \\ and police in the management of information*
}

\section{A incorporação das TIC e sua contribuição para a interacção entre os cidadãos e policiais na gestão da informação*}

\author{
Jorge Ignacio López Gutiérrez** \\ Nohemí Gutiérrez Avendaño***
}

\section{Resumen}

En el presente trabajo se realizó una aproximación teórica a la dimensión de las TIC en el marco de la recolección de información que pueda aportar a la seguridad ciudadana desde la perspectiva del servicio de inteligencia. En ella se contempló una visión desde el ciudadano y desde expertos en la institución sobre el manejo de la información y las TIC. La metodología utilizada fue desde un enfoque cualitativo con la aplicación de dos instrumentos de recolección, entre ellos la encuesta y la entrevista a informante clave, dando como resultado una propuesta de protocolo de entrevista virtual para la recolección de información de fuentes humanas.

Palabras clave: entrevista virtual, fuente humana, recolección de información, seguridad ciudadana.

Fecha de recepción del artículo: 24 de Febrero de 2017

Fecha de aceptación del artículo: 21 de Diciembre de 2017

DOI: https://doi.org//0.22335/rlct.v6i3.373

* Este artículo es producto del proyecto de investigación denominado "Incorporación de tecnologías móviles en la recolección de la información a través de fuentes humanas en la inteligencia policial", realizado dentro del grupo de investigación ESCIC-DINAE y semillero de investigación Día-Logos de la Escuela de Inteligencia y Contrainteligencia de la Policía Nacional. El grupo se encuentra registrado en categoría C Colciencias

** Investigador Principal: Intendente de la Policía Nacional. Técnico profesional en servicio de policía. Estudiante de ingeniería en telecomunicaciones.correo electrónico: escic.arinv@policia.gov.co

*** Co-investigador: Nohemí Gutiérrez Avendaño. Patrullera de la Policía Nacional. Magister en Docencia e Investigación. Especialista en Docencia e Investigación Universitaria. Psicóloga.Técnico profesiona en servicio de policía

\begin{abstract}
In the present work a theoretical approach to the ICT dimension was made in the context of the collection of information that can contribute to citizen security from the perspective of the intelligence service. It saw a vision from the citizen and from experts in the institution on the management of information and ICT.The methodology used was from a qualitative approach with the application of two collection instruments, among them the survey and the key informant interview, resulting in a proposal of a virtual interview protocol for the collection of information from human sources.
\end{abstract}

Keywords: Virtual interview, human source, information gathering, citizen security.

\section{Resumo}

Neste trabalho foi realizada uma abordagem teórica à dimensão das TIC como parte do conjunto de informações que podem contribuir para a segurança pública a partir da perspectiva do serviço de inteligência. Ele contemplou uma visão do cidadão ea instituição de especialistas em gestão de informação e TIC.A metodologia utilizada foi a partir de uma abordagem qualitativa, com a aplicação de dois instrumentos de coleta, incluindo a pesquisa e entrevistas com informantes-chave, resultando em uma entrevista virtual de 
protocolo proposto para a coleta de informações de fontes humanas.

Palavras-chave: entrevista virtual, de origem humana, de recolha de dados, segurança pública.

\section{Introducción}

En la búsqueda incesante de soluciones a las diferentes problemáticas que afrontan las comunidades, se han abordado múltiples herramientas de recolección de información desde la perspectiva cualitativa en investigación, entre ellas la entrevista. Ésta, considerada como una técnica que permite flexibilidad a partir de los objetivos, las funciones y los ámbitos de abordaje, sin embargo, el éxito en la obtención de información basa su fundamento en las habilidades del entrevistador, de allí que se oriente la tendencia del contacto personal con el interlocutor como una forma de intercambio de información desde la dimensión verbal y no verbal.

Si bien es cierto, la entrevista conocida tradicionalmente como la interacción entre dos participantes que cumplen roles diferenciales según lo plantea Perpiñá (20I2) en donde "el entrevistador es reconocido como experto en la materia, es el que pide información y el entrevistado es el que origina la demanda esperando sacar provecho de tal relación, y es quien proporciona la información". Esta concepción está en el marco de la interacción personal, ha llevado a la tomar a los organismos del Estado una referencia de esta técnica como fuente primaria de obtención de datos que garantice el mantenimiento de las condiciones de seguridad y el orden interno.

En tal sentido, la Dirección de Inteligencia Policial a través de su proceso de gestión de conocimiento conocido como el ciclo de inteligencia, determinó una fase denominada recolección y cuyo objetivo es obtener de las fuentes de información el insumo necesario que aporte a la producción de inteligencia y contrainteligencia, haciendo un despliegue de actividades entre las que se destaca la administración de fuentes humanas.

En ella se encuentra descrito el protocolo para la obtención especializada de información a través de la adecuada administración de fuentes humanas que manifiesten la voluntad de suministrar información, que apoye la producción de inteligencia estratégica, operacional y para el servicio, dicha obtención realizada a través de técnicas aplicadas como la entrevista a través de una adecuada formulación de preguntas.

Con el propósito de mejorar la seguridad, rapidez, manejo, acceso, oportunidad y disponibilidad de la información suministrada por fuentes humanas en la inteligencia policial, mediante el diseño de un protocolo de entrevistas y el uso de tecnologías móviles, se adelantó el actual proceso y para ello se identificó el uso actual e impacto de las plataformas dispuestas por la Policía Nacional para interactuar con el ciudadano en materia de recolección de información y a partir de ello se presenta una propuesta para la implementación del protocolo de una entrevista virtual para la Policía Nacional.

En la indagación referente al tema, se encuentra que la entrevista es una de las técnicas de investigación de mayor difusión en las Ciencias Sociales, la cual ha sido definida como: una conversación con una estructura y un propósito (Álvarez-Gayou 2004 citado por Teresa \& Leobardo, 2007), en tal sentido se puede decir que la entrevista como instrumento de recolección, es una de las formas de interacción humana que permite recopilar y profundizar sobre diferentes aspectos sociales, convirtiéndolo en un elemento importante dentro de la construcción social de realidad, vista desde el otro, logrando con esto ser un factor esencial para la configuración perspectiva de procesos de investigación en ciencias sociales, aunque es importante resaltar que para que esta técnica sea efectiva se debe desarrollar fundamentada en las habilidades de comunicación para lo cual se utiliza la conversación (latín "conversatio", acción y efecto de reunirse y dar vuelta, en donde un grupo de personas se reúne para exponer diferentes puntos de vista), con una estructura y un propósito para lo cual el entrevistador debe contar con la idoneidad provista por competencias cognitivas, técnicas, racionales y emocionales (Chabot).

La multiplicidad de fenómenos sociales, ligados a dinámicas delincuenciales, requieren de servicios de Inteligencia se encuentren preparados para una acertada administración de fuentes Humanas interacción entre actores, las ciencias naturales explican los fenómenos mediante leyes causales, a diferencia de las ciencias humanas, que comprenden en base a fines, motivos o valores, la propia 
intencionalidad de la acción del sujeto. (Ursúa, 2004), por lo tanto, una entrevista debe estar orientada a factores intrínsecos y extrínsecos de los individuos, puesto que a partir de estos se puede interpretar en todas las dimensiones su realidad social.

Esta dinámica fenomenológica aumenta la incertidumbre frente a la seguridad, tal como lo describe Salinas (1999) (citado por Huesca \& Ortega, 2009) donde define inseguridad ciudadana como "la condición que sufre una comunidad cuando es privada del libre y pacífico ejercicio de los derechos, donde el temor es producido por las acciones violentas". Frente a la función del Estado de garantizar ambientes seguros y en paz, las instituciones como la Policía Nacional aportan en dicha construcción de escenarios, a través de la ejecución de las diferentes actividades.

Una de éstas que contempla la Ley 1621 del 2013, es la realización de "actividades de inteligencia y contrainteligencia utilizando medios humanos o técnicos para la recolección", constituyéndose en actividades que puedan exponer a los agentes a riesgos frente a su identificación ante personas que manifiesten dar información, así como los altos costos económicos que se generan en los desplazamientos, baja seguridad, rapidez en el manejo, acceso, oportunidad y disponibilidad de los datos y la información.

En la Policía Nacional, el proceso de inteligencia en su fase de recolección de información requiere adelantar diferentes actividades entre ellas la administración de fuentes humanas' a través de la realización de entrevistas, enmarcada en el aseguramiento de los fines esenciales del Estado.

En concordancia con lo anterior, se observan riesgos en el desarrollo de esta actividad, así como altos costos económicos relacionados con el desplazamiento de los agentes que recolectan la información, baja seguridad, lentitud en el manejo, acceso, oportunidad y disponibilidad de los datos y la información.

La actividad de inteligencia compromete la identificación de sus funcionarios frente a personas naturales, que aportan información de manera voluntaria, pero se constituye en un riesgo toda vez que las motivaciones pueden estar

I Fuente humana: persona que de manera voluntaria brinda información. Resolución 0 I446 del 20I4. Manual de inteligencia y contrainteligencia de la Policía Nacional. fundamentadas en el engaño y la posibilidad de desestabilizar la institucionalidad.

Por lo anterior, las Tecnologías de la Información y las telecomunicaciones como actual mecanismo de interacción entre individuos, hace parte de los canales de comunicación más utilizados, de acuerdo con el boletín técnico de prensa publicado el 6 de abril de 2015 por parte del Departamento Administrativo Nacional de Estadística DANE- denominado Indicadores Básicos de Tenencia y Uso de Tecnologías de Información y las Telecomunicaciones - TIC- en Hogares y personas de 5 y más años de edad 2014 se evidencia que: En 2014, para el total nacional, en el 95,3\% de los hogares al menos una persona poseía servicio de telefonía por celular, en 2013 el porcentaje fue de $94,7 \%$, en 2014 , el $38,0 \%$ del total nacional de hogares poseía conexión a Internet, en 2013 el porcentaje fue de $35,7 \%$, en 2014 , el $52,6 \%$ del total nacional de personas de 5 y más años de edad usaba computador.

En razón a lo anterior se planteó el siguiente cuestionamiento: ¿Cómo mejorar la seguridad, rapidez, manejo, acceso, oportunidad y disponibilidad de la información suministrada por fuentes humanas en la inteligencia policial?

La trasformación constante del mundo en el marco de los procesos de globalización se extiende a la información, es así que el término "sociedad de la información" se ha incorporado en el lenguaje de la actualidad, dando sentido a lo citado por Baelo (2008) frente a la reflexión de autores como Aron (1972) y Touraine (1969) en relación con la concepción de "una sociedad post - industrial, en la que la configuración vendría determinada en torno a la información y el conocimiento", esto deja ver la importancia que tiene hoy los modelos de gestión de la información en los entornos del ser humano, para el caso que atañe a la presente investigación, lo relacionado con la información y la seguridad.

En estos procesos de intercambio, la comunicación humana es una herramienta que promueve la transformación de realidades en donde se involucran elementos disponibles para la generación de conocimiento. Pascual (2005) define ésta como "el proceso inevitable y constante entre los actores sociales (todo es comunicación), diversa (verbal y no verbal), contextual (cada intercambio crea su 
contexto de interpretación, y el concepto de contexto se hace dinámico y complejo).

Dicho proceso ha gestado cambios significativos en la historia hasta llegar a lo que hoy se conoce como sociedad de la información, facilitando a través de las tecnologías el alcance a todos los rincones de la geografía, en la actualidad las TIC se han igualado a una revolución "comparable a las suscitadas por la escritura, la imprenta o la industrialización" (Echeverría, 2009). Esta revolución ha posibilitado introducir otras formas de interacción como la modalidad a distancia, en donde se a través de la televisión, el teléfono, la radio, de igual forma la creación de redes usando las comunidades informáticas y el intercambio de información haciendo uso de medios como los correos electrónicos.

Frente a las características representativas de las TIC descritas por Cabero (1998) (Citado por Belloch, 2016), es importante retomar las que se relacionan al presente proyecto de investigación y tienen que ver con las siguientes:

La inmaterialidad, la información puede ser llevada de forma transparente e instantánea a lugares lejanos, la interconexión, hace referencia a la creación de nuevas posibilidades tecnológicas a partir de la conexión entre dos tecnologías, la instantaneidad, es decir las redes de comunicación y su integración con la informática, han posibilitado el uso de servicios que permiten la comunicación y transmisión de la información, entre lugares alejados físicamente, de una forma rápida.

Estas características descritas anteriormente están en el marco de la utilidad del uso de las TIC para el abordaje de la entrevista como una herramienta de recolección de información estandarizada en los procesos de investigación científica y de generación de conocimiento.

Otros antecedentes conceptuales encontrados frente a las TIC, son los descritos por Gil Díaz (1985), quien hace referencia a éstas como "sistemas o productos que son capaces de captar información del entorno, de almacenarla, de procesarla, de tomar decisiones, de trasmirirlas y de hacerlas inteligibles a los sentidos", así como la dada por Adell (1997) y definida como "el conjunto de procesos y productos derivados de las nuevas herramientas (hardware y software), soportes de la información y canales de la comunicación relacionados con el almacenamiento, procesamiento y trasmisión digitalizada de la información" (Citados por Baelo, 2008).

Lo anterior resalta la oportunidad que se gesta con la incorporación de TIC en el manejo de la información ya que se vuelve más viable, rápida y efectiva su recepción, procesamiento y almacenamiento; dichas características enunicadas contribuyen a una mejor comprensión del tema de investigación.

La importancia de las tecnologías dentro de las organizaciones, dan a conocer la gran transcendencia e influencia que hoy poseen en el mundo, dichas tecnologías han disminuido en gran manera las barreras que anteriormente existían para realizar negocios e incrementar los ingresos, $y$ de igual forma aplicar nuevas herramientas que contribuyan al progreso de las compañías ha sido un motor de crecimiento.

Cabe resaltar que hoy en día la implementación de estas herramientas no son una inversión; sino una necesidad, ya que éstas le permiten a las grandes y pequeñas empresas entrar en una etapa de crecimiento y estar a la vanguardia en la actualidad, generando en ellas organizaciones modernas y competitivas con proyección a nivel nacional e internacional.

Martínez (20II) en una reflexión descriptiva sobre el tema, buscó profundizar y conocer el impacto e importancia que poseen las TIC dentro de las empresas, mostrando como la tecnología se ha convertido en una necesidad fundamental a la hora de realizar un negocio e incrementar los ingresos de las organizaciones. Permitiéndole a las mismas un acercamiento y mejor comunicación con sus clientes. Esto se logró determinar gracias a la opinión y amplia experiencia de personas con grandes conocimientos en aplicaciones tecnológicas y crecimiento empresarial, quienes llegaron a la conclusión de que para que una empresa crezca y obtenga excelentes resultados debe estar a la vanguardia de la utilización de herramientas tecnológicas dentro de sus organizaciones.

Los hallazgos de Martínez (20II) identificaron como aspecto relevante que las TIC son una herramienta indispensable y necesaria para que una organización tenga un impacto a nivel nacional e internacional. Ya que 
esto le generara un acercamiento con el mundo que las rodea, proyectándolas a mejorar y a crecer cada día. Es importante dejar claro que para las mismas, el activo más significativo es la información, definiéndola como el recurso clave de las empresas. Por lo tanto, las tecnologías de la información juegan un papel muy importante dentro de las sociedades.

Por otra parte, en el marco del desarrollo de las TIC se han incorporado las denominadas "aplicaciones móviles", y refieren a ellas las que fueron desarrolladas para ejecutarse en dispositivos móviles, los desarrolladores de estos software se han enfocado en los atributos de calidad para los usuarios, entre ellos, uno considerado de gran importancia como lo es la usabilidad, la cual representa "la facilidad con la que un usuario puede usar una aplicación de software" (Enriquez \& Casas, 20l3), de acuerdo al este criterios puede medirse el grado de uso que puede tener en los usuarios finales.

En tal sentido, estos autores incluyen los atributos (características) de una aplicación o sistema de software y su contexto mencionando entre ellos la efectividad, eficiencia, satisfacción, facilidad de aprendizaje, memorabilidad, contenido, accesibilidad, seguridad, portabilidad y el contexto (Enriquez \& Casas, 20l3).

Este marco referencial que está en desarrollo y en crecimiento constante deja claro que las personas acceden desde sus dispositivos a datos, aplicaciones entre otras donde se encuentre ubicado. Es importante sin embargo, tener en cuenta que el manejo de tecnología móvil con estas características requiere de condiciones de conexión (ancho de banda), situación que puede ser variable de acuerdo a la cobertura existente.

La necesidad de la interconexión mundial, mediada por el desarrollo económico del país, favoreció que Colombia a partir del 2009, con la Ley I34I transformó el Ministerio de Comunicaciones en Ministerio de Tecnologías de la Información y las Comunicaciones, dando "acceso y uso de las TIC a través de la masificación, el impulso a la libre competencia, el uso eficiente de la infraestructura y fortaleciendo la protección de los derechos de los usuarios" $\left(\mathrm{MINTIC}^{2}\right)$.

2 Recuperado de http://www.mintic.gov.co/portal/604/w3-propertyvalue-6077.html
El Gobierno Nacional en la necesidad de generar cultura frente a las nuevas tecnologías de la información y la comunicación ha involucrado diferentes planes y programas que han dado resultados positivos tal como lo muestra el informe del Plan vive digital Colombia el cual ha sido reconocido como la mejor política del mundo en el año 2012 , dando paso a la continuidad con la estrategia nacional en el cuatrienio 2014-2018.

El camino a seguir en los próximos años está orientada a desplegar más contenidos y aplicaciones que contribuyan a consolidar un país en paz a través del desarrollo de contenidos y aplicaciones digitales con impacto social.

De acuerdo al informe, los objetivos planteados para el cuatrienio son los siguientes: I. Convertir a Colombia en un líder mundial en el desarrollo de aplicaciones sociales dirigidas a los más pobres y 2. Tener el Gobierno más eficiente y transparente gracias a las TIC.

Esto tiene implicaciones que involucran a las instituciones públicas como la Policía Nacional de Colombia a optimizar herramientas que favorezcan la gestión y el acceso a las clases menos favorecidas, esto redunda en un compromiso con la seguridad frente a la contribución del ciudadano colombiano que brinda información, posibilitando el uso de las TIC y creando línea directa con la institución para atender situaciones reales y particulares para las comunidades.

Para el año 2010 se definió en Colombia un marco conceptual referente al modelo de Gobierno Electrónico, éste fue documentado en el Conpes 3650, donde se definió que el uso de las Tecnologías de la Información y las Comunicaciones (TIC), era una alternativa disponible para "mejorar los servicios e información ofrecidos a los ciudadanos, aumentar la eficiencia y eficacia de la gestión pública e incrementar sustantivamente la transparencia del sector público y la participación ciudadana" (MINTIC, $2010)$.

De igual forma, para el año 2015 la Corporación Colombia Digital destacó a través de página web la implementación de una la aplicación de seguridad ciudadana en Colombia denominada "Suapp". El lugar de desarrollo e implementación fue el municipio de Soacha (Cundinamarca), donde la empresa Soluciones de localización inteligente -

(n) 
Servinformación, puso en ejecución un aplicativo que permite a los ciudadanos realizar denuncios como hurto, homicidios, microtráfico, conductas antiecológicas, entre otros hechos delictivos.

Para el mes de octubre del mismo año, se incorporaron a la plataforma tecnológica I 39 alarmas comunitarias, 450 cámaras de seguridad, y 206 uniformados de la Policía Nacional que rastrean los focos de inseguridad, denunciados a través de la app. El impacto identificado luego de cuatro meses de su implementación, las cifras de delitos como el hurto y el homicidio descendió en un $19 \%$, de acuerdo con información suministrada por la Policía. (Corporación Colombia Digital, 2015)

La Policía Nacional en el marco de la integración de las políticas de TIC, incluyó en el año 2012 la sistematización de todos los procesos misionales, además de la certificación internacional de ciudadano digital, la creación de un correo electrónico institucional para todo el personal activo, así mismo, la implementación de aplicativos que permiten video conferencias entre las regiones de policía y Direcciones, como una herramienta de fortalecimiento de la gestión interna y en los últimos años abriendo espacio en la prestación de un mejor servicio a los ciudadanos a través de herramientas como su página web: www.policia.gov.co, donde se encuentran disponibles espacios para la denuncia ciudadana y la contribución a la seguridad. Por su parte, el desarrollo de aplicaciones tecnológicas que han puesto al ciudadano a un Clic del servicio de policía.

En la actualidad la Policía Nacional frente a la responsabilidad adquirida de garantizar la seguridad y convivencia ciudadana alineándose a las políticas de gobierno en línea, brinda una aplicación tecnológica móvil a nivel nacional denominada "POLIS", la cual está al alcance de la ciudadanía, permitiéndole acceder a un contacto rápido y efectivo para la atención de requerimientos del servicio de policía.

Dicha aplicación móvil facilita la interacción con el ciudadano a través de la incorporación de un botón de pánico el cual una vez se activa ubica a las patrullas de policía en el lugar donde está ocurriendo el hecho delictivo, de igual forma permite la marcación directa a la línea 123 , favoreciendo la atención de ayuda de los organismos de emergencias vinculados al mismo. Finalmente desarrolla un módulo de reporte en línea en donde se pueden registrar documentos perdidos y elementos extraviados, generando al ciudadano una denuncia inmediata.

Teniendo en cuenta lo anterior, la institución policial se vincula desde la tecnología en el impacto hacia la comunidad, abriendo espacios de atención policial diferentes, sin embargo, no se cuenta con una aplicación que permita a los colombianos brindar información con mayor profundidad sobre fenómenos que afecten la seguridad $y$ convivencia de nuestro país

En el desarrollo de la referenciación teórica se exploró el ámbito de la informática y las telecomunicaciones las cuales han permitido avances significativos que dan vida a las TIC, éstas entendidas como "el conjunto de tecnologías que permiten el acceso, producción, tratamiento y comunicación de información presentada en diferentes códigos como texto, imagen, sonido, etc." (Belloch, 20I5).

Para la presente investigación se tomará la definición de Cabrero frente al concepto de tecnologías de la información y comunicación, quien hace un acercamiento $y$ reflexión sobre el tema, así:

...las nuevas tecnologías de la información y la comunicación son las que giran en torno a tres medios básicos: la informática, la microelectrónica y las telecomunicaciones; pero giran, no sólo de forma aislada, sino lo que es más significativo de manera interactiva e interconexionadas, lo que permite conseguir nuevas realidades comunicativas... (Cabrero, 1998)

De igual forma, serán tomadas las características representativas de las TIC retomadas por Belloch (20I5), las cuales servirán como marco de referencia desde las categorías teóricas que favorecerán el análisis posterior. De ellas se describirán las siguientes:

I. Inmaterialidad. Las TIC permiten la creación, el proceso y la comunicación de la información. Esta información es básicamente inmaterial y puede ser llevada de forma transparente e instantánea a lugares lejanos.

2. Innovación. Las TIC están produciendo una innovación y cambio constante en todos los ámbitos sociales, produciendo simbiosis con otros medios disponibles como la correspondencia personal, dando uso y potencialidades del correo electrónico. 
3. Tendencia hacia automatización. Refiere a la aparición de diferentes posibilidades y herramientas que permiten un manejo automático de la información en diversas actividades personales, profesionales y sociales. La necesidad de disponer de información estructurada hace que se desarrollen gestores personales o corporativos con distintos fines y de acuerdo con unos determinados principios.

4. Diversidad. La utilidad de las tecnologías puede ser muy diversa, desde la mera comunicación entre personas, hasta el proceso de la información para crear informaciones nuevas.

5. Interactividad. La interactividad es posiblemente la característica más importante de las TIC para su aplicación. Mediante las TIC se consigue un intercambio de información entre el usuario y el ordenador. Esta característica permite adaptar los recursos utilizados a las necesidades y características de los sujetos, en función de la interacción concreta del sujeto con el ordenador.

6. Interconexión. La interconexión hace referencia a la creación de nuevas posibilidades tecnológicas a partir de la conexión entre dos tecnologías. Por ejemplo, la telemática es la interconexión entre la informática y las tecnologías de comunicación, propiciando con ello, nuevos recursos.

7. Instantaneidad. Las redes de comunicación y su integración con la informática, han posibilitado el uso de servicios que permiten la comunicación y transmisión de la información, entre lugares alejados físicamente, de una forma rápida.

8. Elevados parámetros de calidad de imagen y sonido. El proceso y transmisión de la información abarca todo tipo de información: textual, imagen y sonido, por lo que los avances han ido encaminados a conseguir transmisiones multimedia de gran calidad, lo cual ha sido facilitado por el proceso de digitalización.

9. Digitalización. Su objetivo es que la información de distinto tipo (sonidos, texto, imágenes, animaciones, etc.) pueda ser transmitida por los mismos medios al estar representada en un formato único universal. En algunos casos, por ejemplo los sonidos, la transmisión radicional se hace de forma analógica y para que puedan comunicarse de forma consistente por medio de las redes telemáticas es necesario su transcripción a una codificación digital, que en este caso realiza bien un soporte de hardware como el MODEM o un soporte de software para la digitalización.
Por otra parte, se retomaron los conceptos en la comunicación mediada por TIC, como los denominados comunicación asincrónica y sincrónica, los cuales nos ayudan a entender la dinámica del proceso que puede establecerse a través de Tecnologías móviles.

La comunicación asincrónica es entendida como aquella que no puede establecerse en tiempo real, como por ejemplo el correo electrónico, ya que este lo que permite es enviar y recepcionar información personalizada a través del intercambio de mensajes entre usuarios que estén conectados a internet pero no interactuando de manera simultánea. Las ventajas atribuidas a este tipo de comunicación son la "rapidez, comodidad, economía, posibilidad de archivos adjuntos" (Valberde, Recuperado en Marzo 20l6)

A su turno, Valverde (Recuperado en Marzo 2016) también define la comunicación sincrónica como aquella que puede establecerse en tiempo real, como un ejemplo define las audioconferencia-videoconferencia, en donde un especialista en un tema puede pronunciar una conferencia que puede ser escuchada y visionada por un grupo de interlocutores, situados en diferentes lugares. Ha sido incorporada exitosamente en ambientes educativos donde la videoconferencia permite una mejor aproximación a la enseñanza presencial dentro del "aula", sustituyendo este espacio físico por el "aula virtual" de la que forman parte todos los participantes en la videoconferencia.

Este autor relaciona también como herramienta útil en la conectividad en tiempo real, el IRC (Internet Relay Chat) o también conocido "chat" el cual permite realizar conversaciones a través de Internet. Como característica relevante en este tipo de comunicación es que los usuarios que utilicen IRC, "deben estar conectados al mismo tiempo dentro de un canal”.

Para Burke y Miller (200l, citado por Castañeda \& Jiménez, 2007), introducen otra herramienta de comunicación mediada por TIC como lo es la técnica de entrevista a través del teléfono, éstos sostienen que esta técnica "facilita la obtención de datos de tipo cualitativo...proporcionando información relevante al conocimiento peculiar de las personas sobre determinado suceso o evento". Referencian que es una técnica de mucha utilidad cuando se requiere de testimonios verbales donde se exprese sobre la participación o haber presenciado un acontecimiento o 
hecho. La cualidad que más resaltan de esta modalidad de entrevista es que la recolección de información se puede realizar desde lugares dispersos y de forma rápida.

En relación con lo que debe contener la entrevista y sus fases, se realizó un proceso de selección de la literatura que contuviese elementos que incorporaran características que pueden ser mediadas por TIC, por ejemplo, lo descrito por Navarro (20l I), donde refiere la importancia de observar con atención, entendida ésta como la observación coordinada (consciente) para interpretar con éxito a la gente y detectar sus indicios no verbales.

De igual forma, el observar dentro del contexto es clave para comprender el comportamiento no verbal, después de un accidente las personas sufren bloqueos mentales, es normal que al comienzo de una entrevista se manifieste nerviosismo y este desaparece mientras transcurre la entrevista, si el comportamiento nervioso se vuelve a presentar se deben plantear preguntas específicas, el entrevistador debe preguntarse por qué surge.

Otro aspecto mencionado es aprender a reconocer y a descifrar los comportamientos no verbales que son universales, establecer una línea base de comportamientos de una persona, posturas iniciales, comprender los comportamientos no verbales idiosincrásicos, observar patrones de comportamiento de las personas con las que nos relacionamos (amigos, familia, compañeros de trabajo, proveedores etc.).

Esta identificación y comprensión intenta buscar en los demás comportamientos gestuales que ocurran a la vez 0 de forma consecutiva, observar si hay alteraciones en el comportamiento de la persona que puedan indicar cambios en sus pensamientos, sus emociones, su intereses 0 su intención, los cambios repentinos de comportamiento pueden ayudar a revelar como una persona esta procesando la informacion o com se esta adaptando a los acontecimientos emocionales.

Navarro (20II) tambien menciona que es fundamental aprender a detectar señales no verbales erroneas o falsas, saber como distinguir entre el bienestar y el malestar, lo cual ayudará a concentrarte en los comportamientos mas importantes para descifrar la comunicación no verbal. La consideración que enfatiza es que cuando se observe a los demás, debe hacerse con la discrecion posible, evitando que el otro pueda darse cuenta y modificar su conducta no verbal.

Por su parte, Olaz (2004) en su libro "La entrevista en profundidad: justificación metodológica y guía de actuación práctica", describe las cuatro fases que debe contener una entrevista y donde a cada una asigna una tarea particular. La primera denominada contacto inicial, la segunda la apertura, la fase de desarrollo y la fase de cierre.

Perpiña (20I2) por su parte, describe de igual manera unas etapas para el desarrollo de las entrevistas, las cuales se vinculan la apertura, el desarrollo y el cierre, dando como punto central en la etapa de desarrollo la exposición y la solución de las objeciones.

Otra visión de la entrevista la da Sánchez (2003) desde una perspectiva periodística, realiza unos planteamientos frente a las fases entre las que describe la aproximación, como aquella donde interviene la simpatía de cada cual, la experiencia y el acierto en el modo de presentarse, recomienda para ello utilizar el "usted" en el tratamiento hacia el entrevistado, explicar al entrevistado cual es el objetivo y para que se utilizará la información que el entrevistado brinde, mostrar competencia en el conocimiento del tema tratado, dar la impresión del dominio del tema sin parecer ostentoso, tener en cuenta el "off record", cuando el entrevistado no quiere que alguna opinión sea publicada tener en cuenta la actitud, no ser agresivo o intransigente, utilizar un ligero cabeceo para denotar que sigue al entrevistado, es importante cuidar el lenguaje no verbal, evitando ojear papeles mientras el otro contesta, mantener siempre la vista en el interlocutor cuando se pregunta y cuando se contesta y no interrumpir al entrevistado, salvo que se advierta que se distancia del tema estar pendiente de las preguntas $y$ formular nuevas. Finalmente, al cierre de la entrevista revisar y verificar la información, cotejando cifras que no han sido anotadas, nombres, fechas y demás datos relevantes que se consideren.

El libro la entrevista de trabajo (Puchol, 2006) desarrolla de igual forma las diferentes etapas que pueden adelantarse durante una entrevista, describiendo los elementos relevantes para su culminación exitosa, entre ellas denomina la etapa introductoria, la etapa central y la etapa final o cierre de la entrevista. 
El desarrollo teórico se enmarca en los atributos de las TIC y la entrevista como herramientas que pueden integrarse, sin dejar de cumplir sus propósitos originales de recolectar información para la toma de decisiones. La entrevista como forma diferencial frente a la gestión de la información el marco las diferentes etapas para su ejecución, siendo las TIC un facilitador.

\section{Materiales Y Métodos}

Esta propuesta de investigación se fundamenta teóricamente desde la postura epistemológica del pragmatismo, descrita por Peirce y James en 1975, quienes señalaban que "la producción del conocimiento debe comenzar con los problemas prácticos” (Hurtado, 20I0). En tal sentido, permite descubrir desde la realidad cercana, aquellas situaciones susceptibles de solucionar a partir de las acciones que mejoren las condiciones actuales de una comunidad o sector.

La muestra poblacional fue intencional, toda vez que se seleccionaron personas de una comunidad en la localidad de Tunjuelito de la ciudad de Bogotá y Expertos en el manejo de TIC y administración de fuentes humanas en la inteligencia policial, quienes cumplían características relacionados a nuestro tema de interés.

Como instrumentos para la recolección fueron utilizados la Entrevista a informante clave y la encuesta. La primera descrita por Buendía, Colás \& Hernández (200I) (citados por Bernal, 20l4), definiéndola como un instrumento para "recoger información mediante un proceso directo de comunicación entre entrevistadores y entrevistados en el cual se responden cuestionamiento previamente diseñadas en función de las dimensiones que se pretenden estudiar, planteadas por el entrevistador".

El informante clave es una persona que por sus vivencias, capacidad de empatizar y relaciones que tienen en el campo pueden apadrinar al investigador convirtiéndose en una fuente importante de información a la vez que le va abriendo el acceso a otras personas y a nuevos escenarios (Robledo, 2009) ${ }^{3}$.

3 Juana Robledo Martín Nure Investigación, $n^{\circ}$ 42, Septiembre - Octubre 09.
Por otro lado, la encuesta es una búsqueda sistemática de información en la que el investigador pregunta a los investigados sobre los datos que desea obtener, y posteriormente reúne estos datos individuales para obtener durante la evaluación datos agregados. Como característica primordial es realizada a todos los entrevistados con las mismas preguntas, en el mismo orden, y en una situación similar; de modo que las diferencias son atribuibles a las diferencias entre las personas entrevistadas (Escobar \& Bonilla) ${ }^{4}$.

\section{Resultados $Y$ Conclusiones}

De acuerdo con la información recolectada y analizada, se puede decir que existe viabilidad para incorporar las tecnologías móviles en la recolección de información de fuentes humanas teniendo en cuenta que:

Un gran porcentaje representado en el $74 \%$ utiliza teléfonos con tecnología Smartphone y la frecuencia en su utilización es del $83 \%$ (100). Por otra parte, un $72 \%$ cuenta con servicio de internet en su celular, indicando con ello que hace uso de las diferentes herramientas tecnológicas disponibles.

En importante resaltar que la cifra es coherente con el número de personas encuestadas ya que del $74 \%$ de ellos manifestó tener teléfonos celulares con tecnología Smartphone, solo un $2 \%$ no tendría acceso a internet desde su teléfono.

De igual forma, existe poco conocimiento de aplicaciones de celular para comunicarse con la Policía Nacional, toda vez que tan solo un $9 \%$ (II) de los encuestados manifestó conocer alguna. Por otra parte, el 91\% (109) no ha escuchado ni conoce aplicaciones de comunicación con la Policía. De las aplicaciones que el $9 \%$ de las personas mencionaron conocer, hicieron alusión a "polis" (8) y cuadrante amigo (2).

Teniendo en cuenta que el uso de aplicaciones móviles es la posibilidad contemplada en el intercambio de información entre el ciudadano y la Policía Nacional, es considerable el porcentaje de las personas que no conoce

4 Jazmine Escobar I y Francy Ivonne Bonilla-Jiménez, Cuadernos Hispanoamericanos de Psicología,Vol. 9 No. I, 5 I-67 
esta información, por ello es necesario pensar en una estrategia de difusión por parte de la Policía Nacional que permita mejorar la apropiación de las comunidades.

Por otra parte, en la actualidad no se brindan las condiciones de generación de confianza con el ciudadano ya que un $\mathbf{8 5 \%}$ de las personas encuestadas indican que prefieren no vincular a la policía en la solución de problemas por razones como: "que las patrullas no llegan al requerimiento o no contestan las líneas", otros manifiestan que prefieren llamar a la línea dispuesta en el cuadrante 0 ir personalmente al CAI más cercano, esta consideración debe ser objeto de reflexión toda vez que la cercanía con el usuario es un elemento contextual importante en referencia a los niveles de usabilidad de una aplicación móvil desarrollada para la seguridad.

En relación con la mejoraría de la seguridad a través de la interacción entre el ciudadano y la Policía Nacional. El $84 \%$ (IOI) de los encuestados tiene una consideración positiva de la mejora de la seguridad a través de una APP que permita dicha interacción. Entre las consideraciones que plantean los ciudadanos aducen que ello permitirá ampliar los canales de comunicación con la comunidad, podrá ser de ayuda ya que la inseguridad siempre va existir y serviría para opinar sobre el servicio, así como volverlo más efectivo.

Otra dimensión contemplada en el proceso de investigación es la cobertura de los servicios de la Policía Nacional al interactuar con los ciudadanos a partir de una aplicación móvil, frente a este ítem existe un $79 \%$ de aceptación teniendo en cuenta las condiciones actuales de uso de tecnologías y acceso de internet, dejando como aspecto relevante la oportunidad de ser escuchados como ciudadanos y de la oportunidad en la atención.

En lo referente a las herramientas tecnológicas que garanticen la seguridad, se da importancia a la incorporación de tecnologías que favorezcan una comunicación más eficiente entre ciudadano y policía, teniendo en cuenta la tendencia creciente de usuarios con dispositivos móviles conectados a internet, favoreciendo con ello la interconexión y la convergencia tecnológica para garantizar ciudades más seguras.
Se hace necesario para ello determinar una la plataforma multimedia que permita dar una mejor interacción, de acuerdo a las necesidades de seguridad y favoreciendo la administración de información dada por el ciudadano. Debe plantearse en la reflexión sobre la articulación técnica con el talento humano necesario para lograr tener la capacidad de respuesta esperada.

Finalmente, de acuerdo a la revisión documental y las mesas de trabajo con expertos se realizan las consideraciones frente al perfil del entrevistador en el contexto de inteligencia policial como una persona que cuente con la experiencia necesaria en el servicio en la administración de fuentes humanas, con bases conceptuales y prácticas sobre la aplicación de técnicas de entrevista, interpretación de lenguaje no verbal y dominio en herramientas ofimáticas que le permitan interactuar efectivamente con el ciudadano.

En relación con la identificación de la plataforma tecnológica accesible, vigente y segura para la aplicación que permita la recolección de información, se utilizó la entrevista a informante clave quien realizó aportes frente a la forma adecuada de recolectar información del ciudadano sobre aspectos que afecten su seguridad, mencionando que deben utilizarse estructuras de información, saber que la información que se recolecte pueda servir, es necesario determinar variables de recolección y poder definir criterios para categorizarla con el fin de obtener un beneficio.

En cuanto a hardware y software, la consideración realizada frente a la forma más apropiada de administrar información dada por los ciudadanos en cuanto a la seguridad, es importante establecer un alcance, a qué población va a estar dirigido el esfuerzo institucional, es necesario tener en cuenta el sistema operativo de los dispositivos móviles (IOS, ANDROID, etc). Un argumento frente a usuarios potenciales lo refiere a los funcionarios de policía en uso de buen retiro, quienes pueden considerase como fuentes a futuro. Frente la información debe considerarse que tipo será almacenado, si audio, video, texto ya que de acuerdo a ello se pueden vincular protocolos en https, json, webservice, resfull.

En lo referente a las herramientas tecnológicas que garanticen la seguridad de la información sobre aspectos que 
afecten la seguridad ciudadana, brindada por los ciudadanos, es imprescindible considerar que la aplicación móvil deba descargarse desde sitios seguros como Appstore, google play o directamente de la página web de la Policía Nacional. La incorporación de este tipo de herramientas en un futuro podría ser el medio de comunicación más eficiente entre ciudadano y policía, argumentando que según estadísticas para el año 2020, estarían conectados ocho billones de dispositivos móviles desde el proceso evolutivo de la tecnología, la interconexión que permite, el concepto de ciudades inteligentes, la convergencia tecnológica.

Por otra parte, relacionando las aplicaciones móviles disponibles en el mercado relacionadas con seguridad ciudadana, se realiza la consideración que la más aceptada para el uso cotidiano de un ciudadano en la actualidad es POLIS, ya que será la aplicación móvil con la que se centraliza la atención virtual de los ciudadanos a nivel nacional. Esta aplicación cuenta con más de 200.000 descargas lo que referencia una tendencia positiva de crecimiento y de usabilidad. Como referente debe tenerse en cuenta que de las 200.000 descargas hay un aproximado del $15 \%$ y $20 \%$ en usabilidad, en razón a que en la actualidad la aplicación POLIS fue creada exclusivamente para atender emergencias

Se planteó de igual forma las características a tenerse en cuenta para desplegar una aplicación llamativa al ciudadano que cuenta con un conocimiento básico de uso de un celular, por ello debe ser práctica, fácil y sencilla, así mismo debe mejorar la cobertura del servicio de policía a través del uso de la aplicación tecnológica, siempre que se realice una buena difusión, haciendo uso de las tecnologías existentes.

Debe entonces determinarse la plataforma multimedia que permita dar una mejor interacción con otras, determinando las necesidades y favoreciendo la administración de información dada por el ciudadano, debe plantearse en la reflexión la articulación técnica con el talento humano necesario para lograr tener la capacidad de respuesta esperada.

Finalmente, esto permite la formulación de una propuesta para la implementación del protocolo de una entrevista virtual de inteligencia en el Servicio de Inteligencia Policial cuyo objetivo se orienta a recolectar información de interés para el servicio de inteligencia policial, a través de una metodología práctica, dinámica y virtual, contribuyendo con la reducción en los costos y riesgos de los funcionarios de inteligencia que buscan anticipar conductas criminales y/o delincuenciales en el territorio Colombiano.

Es importante resaltar que la integración de las tecnologías móviles en los procesos de recolección de información plantean la evaluación de las diferentes formas de comunicación desde el contexto sincrónico y asincrónico, toda vez que permiten la interacción con el usuario ya sea en tiempo real o no. Dicho protocolo incluye el perfil del entrevistador, los criterios para la interacción por videoconferencia, la interacción por mensaje de texto, la interacción por chat en línea y finalmente entrevista telefónica.

I. Interacción por videoconferencia, con las tres fases contempladas, la fase inicial, de desarrollo aplicando las técnicas de entrevista y verificando la información y finalmente la fase de cierre.

2. Interacción por chat en línea y la interacción por mensaje de texto, dando inicio al proceso de rapport y generación de confianza, continuando con el desarrollo de preguntas para recolectar la información brindada por la persona, $y$ finalizando con la intención de generar encuentros posteriores para ampliación de la misma.

3. Entrevista telefónica, teniendo en cuenta los tiempos y la recepción de la información donde solo se tiene la posibilidad de evaluar lo escuchado en el marco del lenguaje no verbal en la categoría de lo paralingüístico para garantizar la confiabilidad de quien brinda la información, de igual forma activando las fases de una entrevista en sus fases de inicio, desarrollo y cierre.

En un mundo globalizado donde las Tecnologías de la Información y las Telecomunicaciones han sido un nodo de interacción entre seres humanos, las organizaciones de seguridad de los Estados deben hacer uso de éstas para poder realizar una efectiva administración de la información, a partir de un uso eficiente de estos recursos tecnológicos tendientes a logro de sus objetivos.

En una realidad como la nuestra, donde los escenarios y las dinámicas actuales están asociadas a los acuerdos de paz, producto de la finalización de un conflicto de más de 50 años, es preponderante el acercamiento de las instituciones del Estado hacia los ciudadanos, logrando con esto conocimiento de su realidad, para así formular 
estrategias efectivas que puedan aportar al cumplimiento de sus expectativas en materia de seguridad y convivencia.

\section{Referencias}

Baelo, Á. R. (Julio de 2008). Tesis Doctoral. Integración de las TIC en los centros de educación superior de Castilla y León. España: Universidad de León.

Bautista, A., \& C,A. (1997). ¿Qué es Tecnología Educativa?:Autores y significados. Revista Píxel-bit.

Belloch, O. C. (2 de Febrero de 20I5). Las tecnologías de la información y comunicación (T.I.C.). Obtenido de Unidad de Tecnología Educativa. Universidad de Valencia: Recuperado de http://www.uv.es/ bellochc/pdf/pwticl.pdf

Bernal, C.A. (20I4). Metodología de la investigación. Pearson.

Cabrero, J. (1998). Impacto de las nuevas tecnologías de la información y la comunicación en las organizaciones educativas. Granada: Grupo Editorial Universitario.

Castañeda, D., \& Jiménez, L. e. (2007). La entrevista telefónica. Estudio sobre las culturas contemporaneas.

Echeverría,J. (2009).Apropiación social de las tecnologías de la información y la comunicación. Red Revista Iberoamericana de Ciencia,Tecnología y Sociedad.

Enriquez, J. G., \& Casas, S. I. (20|3). Usabilidad de las aplicaciones móviles. ICT-UNPA.
Huesca, G. A., \& Ortega, A. E. (2009). La percepción de inseguridad en Madrid. España: Universidad Pontificia Comillas. Recuperado el 2016

Martínez, J. (20I I). Impacto de las TIC en la comunicación corporativa e institucional. Obtenido de Universidad Complutense de Madrid: ProQuest ebrary.

MINTIC. (2010). Documento CONPES 3650. Importancia estratégica de la estrategia de Gobierno en Línea. Bogotá: Departamento de Planeación Nacional.

Navarro, J. (20I I). El cuerpo habla. Málaga: Sirio, 2a edición.

Olaz,A. (2004). La entrevista en profundidad: justificación metodológica y guía de actuación práctica. En A. Olaz, La entrevista en profundidad: justificación metodológica y guía de actuación práctica.

Pascual, R. (2005). Fundamentos de la comunicación humana. España: ECU. Recuperado el 2 de Febrero de 2016

Perpiñá, C. (2012). Manual de la entrevista psicológica: saber escuchar, saber preguntar. España: Larousse-Ediciones Pirámide. Recuperado el 27 de Enero de 2016

Puchol, L. (2006). La entrevista de trabajo. Ediciones Díaz de Santos.

Sánchez,J. F. (2003). Entrevistas Periodísticas: introducción práctica. S.A. EUNSA. Ediciones Universidad de Navarra.

Valberde, B. J. (Recuperado en Marzo 2016). Herramientas de comunicación sincrónica y asincrónica. Educar en red. Internet como recurso para la educación. 